\title{
Breathless or breathtaking: Respiratory motion correction
}

\author{
Dominik C. Benz, MD, a and Tobias A. Fuchs, $M^{a}$ \\ a Department of Nuclear Medicine, University Hospital Zurich, Zurich, Switzerland
}

Received Jul 2, 2016; accepted Jul 5, 2016

doi:10.1007/s12350-016-0590-4

\section{See related article, pp. 419-428}

Please do not move, take a deep breath, and hold it for the next minutes. This would result in an absolute respiratory and patient motion-free myocardial perfusion imaging (MPI) SPECT acquisition-or in a breathless patient.

Thanks to substantial refinements over the past decade MPI SPECT has evolved remarkably and represents a well-established method to assess diagnosis and prognosis in patients with coronary artery disease. However, artificial defects, loss of resolution, image blurring, or even noninterpretable datasets have been described due to patient motion (PM) and respiratory motion (RM). ${ }^{1-3}$ Although motion has been recognized as a potential source of error, the optimal approach to reduce the occurrence and extent of such artifacts has yet to be found.

A revolutionary milestone in the era of SPECT MPI, with the introduction of latest generation cadmium-zinc-telluride (CZT) semiconductor detectors technology and a heart-focused design with optimized image reconstruction software, ${ }^{4}$ has not only resulted in improved count sensitivity and spatial resolution, but has also paved the way for innovative protocols with drastically reduced image acquisition time ${ }^{5}$ or lower radiation dose. ${ }^{6-8}$ This has stimulated research on promising methods for the elimination of motion either prospectively or retrospectively. Motion tracking software $^{9-11}$ or prospective respiratory triggering during inspiration breath-hold ${ }^{12-14}$ has been reported to

Reprint requests: Tobias A. Fuchs, MD, Department of Nuclear Medicine, University Hospital Zurich, Ramistr 100, 8091 Zurich, Switzerland; tobias.fuchs@usz.ch

J Nucl Cardiol 2018;25:429-32.

1071-3581/\$34.00

Copyright (C) 2016 American Society of Nuclear Cardiology. eliminate motion. Although the clinical value of the latter has been repeatedly demonstrated, ${ }^{12-14}$ such an approach inevitably complicates and prolongs acquisition protocols. Therefore, implementation of retrospective respiratory gating might represent a more adequate strategy for controlling RM in daily clinical routine and has recently been reassured by studies that validated different motion detection and correction algorithms for CZT cameras successfully. ${ }^{9-11}$ Notwithstanding the accuracy and reliability of these algorithms, their impact on diagnostic accuracy is yet unclear.

In the current issue of the Journal of Nuclear Cardiology, van Dijk et al. ${ }^{19}$ investigated motion of patients undergoing CZT MPI SPECT in order to determine the value of an automatic motion detection and correction software (MDC for Alcyone, GE Healthcare). This commercially available software retrospectively bins list-mode data from 5 pinhole projections and reconstructs five dynamic images from 5 central pinholes. Five virtual lines are drawn through the intersection of the myocardium center of mass and each of the five pinholes. The point with the smallest distance from these lines is calculated (in the $\mathrm{x}, \mathrm{y}$ and $\mathrm{z}$ dimension) for each bin. Finally, the software tracks motion by comparing all points and generates a system matrix that incorporates the identified motion. In order to validate this software, the authors performed a phantom test that demonstrated an accurate detection of $\mathrm{RM}>2 \mathrm{~mm}$ and $\mathrm{PM}>1 \mathrm{~mm}$. In their retrospective study, 83 consecutive patients with intermediate anatomical coronary lesions who underwent stress/rest ${ }^{99 m}$ Tc-tetrofosmin SPECT MPI were included. Remarkably, FFR measurement by invasive coronary angiography was performed in all patients. A fixed scan time of $8 \mathrm{~min}$ for stress acquisition was used, and data were acquired in list-mode. Attenuation correction was not performed in this study. For RM and PM detection, emission data were reformatted into $1 \mathrm{~s}$ and $20 \mathrm{~s}$ time bins. Nonmotion-corrected scans were compared with corrected scans by qualitative (visual) and semiquantitative (total perfusion deficit [TPD]; 


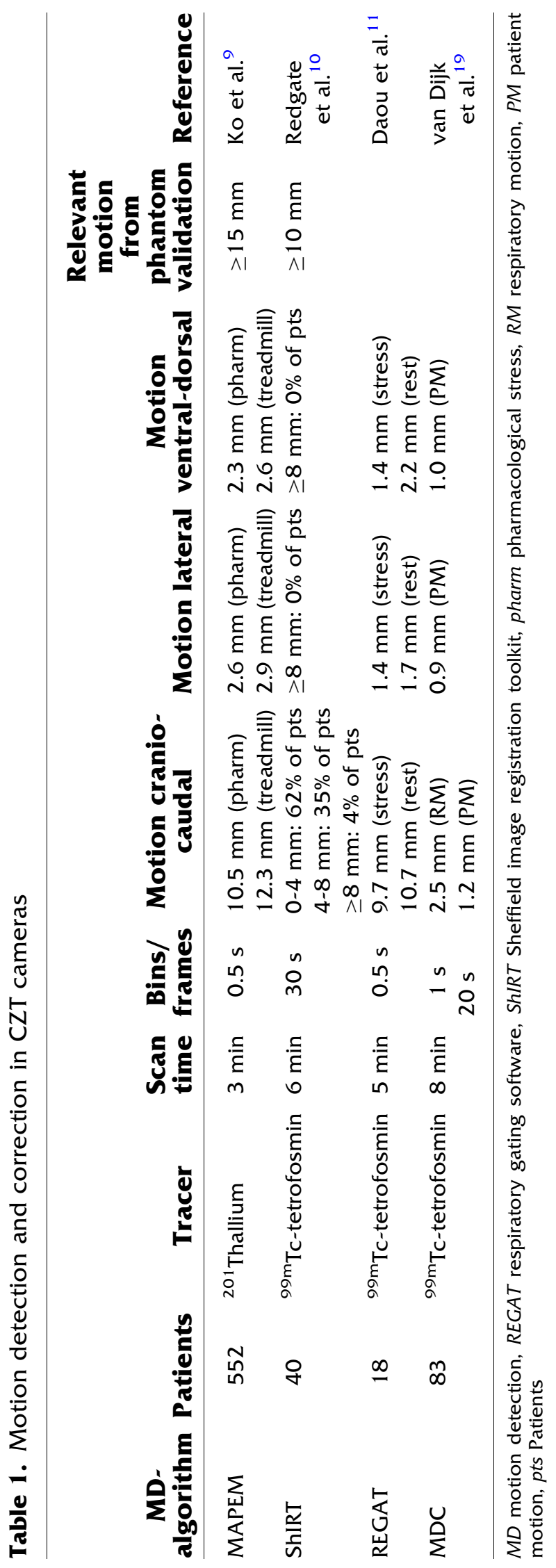


segmental uptake) parameters. If diagnostic outcome changed due to motion correction, the results were compared to FFR results.

In their study, the mean RM was $2.5 \pm 0.4 \mathrm{~mm}$ and the maximum $\mathrm{PM}$ was $2.4 \pm 0.8 \mathrm{~mm}, 2.8 \pm 0.9 \mathrm{~mm}$, and $3.4 \pm 1.5 \mathrm{~mm}$ in the lateral, ventral-dorsal and cranial-caudal direction, respectively. Of note, the mean RM decreased during the scan, especially during the first 2 min. Changes regarding the visual interpretation occurred in nine patients $(11 \%)$ after applying the RM correction algorithm. However, based on FFR as standard of reference, this resulted in deterioration of the diagnostic outcome of five of these nine patients $(6 \%$ of the total study population). A TPD change $\geq 7 \%$ occurred in two patients (3\%) after RM correction was applied and resulted in one patient having improvement and another having deterioration based on FFR. A segmental uptake change $\geq 5 \%$ occurred in 57 patients $(69 \%)$ and resulted in an improvement according to FFR in 30 patients, but resulted in deterioration of 15 patients and remained unknown in 12 patients. The patient motion correction did not change diagnostic outcomes in any of the patients based on SPECT interpretation or TPD. However, based on $\geq 5 \%$ segmental change, the segmental uptake values were corrected in seven patients. Finally, the amount of motion correction and the mean RM or PM did not correlate.

This study adds an important piece to the puzzle of detecting and correcting motion in MPI SPECT. With the introduction of CZT cameras, the application of motion detection and correction algorithms has lately regained widespread interest. ${ }^{10,11}$ The authors should be congratulated for this comprehensive study investigating a commercially available software to automatically detect and correct motion. The study is well conducted, and the results are clearly presented. One of the strengths of the study is the use of invasive FFR as the standard of reference. Nevertheless, it is arguable whether invasive FFR is the ideal standard of reference since concordance between SPECT and invasive FFR has previously been shown to be modest, ${ }^{15,16}$ even in the same study population. ${ }^{17}$ Moreover, the authors' effort to validate the software tool with a basic phantom test adds fundamental background information on its accuracy. The finding that insufficient count statistics might lead to a deterioration of image quality is problematicparticularly in view of the projected steps to decrease radiation exposure through lowering isotope doses and prolonging acquisition times. ${ }^{6}$ As the authors state themselves, the motion detected by MDC in this study, especially RM, was substantially smaller than reported in previous studies. ${ }^{9-11}$ In consideration of the mean RM and PM of $2.5 \mathrm{~mm}$ and $1.2 \mathrm{~mm}$, the authors conclude that correction of this small motion did not appear to improve the diagnostic outcomes, and hence the value of applying motion correction seems limited in MPI CZT SPECT. Based on visual interpretation, this seems to be entirely true in this study population as the SPECT interpretation, after applying the motion correction algorithm, changed in $11 \%$ of patients of whom $44 \%$ resulted in an improvement and $56 \%$ resulted in deterioration. However, a segmental uptake change $\geq 5 \%$ occurred in $69 \%$ of the study population. Furthermore, in addition to the above-mentioned comment on FFR as the standard of reference, this needs to be put in the perspective of recently published work on that topic (Table 1). The scan duration seems to play an important role regarding motion. While scan duration lasted between 3 and $6 \mathrm{~min}$ in the previously reported studies, ${ }^{9-11}$ it was $8 \mathrm{~min}$ in this study. The higher detected motion in previous studies seems in line with the important finding of van Dijk et al. ${ }^{19}$ that RM decreased significantly during the scan, especially in the first $2 \mathrm{~min}$ (e.g., motion was highest during the first $2 \mathrm{~min}$ ). This might suggest that shorter scan protocols-which have become feasible due to CZT detectors - might be more susceptible for motion as the first minutes of excessive movement have a higher relative impact, and therefore motion detection and correction algorithms may play an even more important role in short acquisition protocols. Of course, prolonged scan durations as used in conventional SPECT will again increase the likelihood of motion artifacts. ${ }^{18}$ Explanations, such as longer time bins and higher myocardial count rates, have been discussed in the study of van Dijk et al. ${ }^{19}$ Additionally, the type of stress (pharmacological vs treadmill) seems to play a role as Ko et al. have reported a significant higher motion along the cranio-caudal axis in patients who have undergone SPECT MPI after treadmill stress. ${ }^{9}$

As emphasized by the authors, comfortable environment and sufficient patient information are mandatory principles to reduce motion, but it remains questionable that this will be sufficient in all circumstances. Whether latest technologies for motion detection and correction are a breathtaking breakthrough or whether breathless patients will be the future still seems to require further investigations.

\section{References}

1. Friedman J, Berman DS, Van Train K, Garcia EV, Bietendorf J, Prigent F, et al. Patient motion in thallium-201 myocardial SPECT imaging. An easily identified frequent source of artifactual defect. Clin Nucl Med 1988;13:321-4.

2. Germano G, Chua T, Kavanagh PB, Kiat H, Berman DS. Detection and correction of patient motion in dynamic and static myocardial SPECT using a multi-detector camera. J Nucl Med 1993;34:1349-55. 
3. Cooper JA, Neumann PH, McCandless BK. Effect of patient motion on tomographic myocardial perfusion imaging. J Nucl Med 1992;33:1566-71.

4. Buechel RR, Gaemperli O. Newer generation cameras are preferred. J Nucl Cardiol 2016. doi:10.1007/s12350-016-0462-y.

5. Herzog BA, Buechel RR, Katz R, Brueckner M, Husmann L, Burger IA, et al. Nuclear myocardial perfusion imaging with a cadmium-zinc-telluride detector technique: optimized protocol for scan time reduction. J Nucl Med 2010;51:46-51.

6. Einstein AJ, Blankstein R, Andrews H, Fish M, Padgett R, Hayes $\mathrm{SW}$, et al. Comparison of image quality, myocardial perfusion, and left ventricular function between standard imaging and single-injection ultra-low-dose imaging using a high-efficiency SPECT camera: the MILLISIEVERT study. J Nucl Med 2014;55:1430-7.

7. Benz DC, Templin C, Kaufmann PA, Buechel RR. Ultra-low-dose hybrid single photon emission computed tomography and coronary computed tomography angiography: a comprehensive and non-invasive diagnostic workup of suspected coronary artery disease. Eur Heart J 2015;36:3345.

8. Gimelli A, Bottai M, Genovesi D, Giorgetti A, Di Martino F, Marzullo P. High diagnostic accuracy of low-dose gated-SPECT with solid-state ultrafast detectors: preliminary clinical results. Eur J Nucl Med Mol Imaging 2012;39:83-90.

9. Ko CL, Wu YW, Cheng MF, Yen RF, Wu WC, Tzen KY. Datadriven respiratory motion tracking and compensation in CZT cameras: a comprehensive analysis of phantom and human images. J Nucl Cardiol 2015;22:308-18.

10. Redgate S, Barber DC, Fenner JW, Al-Mohammad A, Taylor JC, Hanney MB, et al. A study to quantify the effect of patient motion and develop methods to detect and correct for motion during myocardial perfusion imaging on a CZT solid-state dedicated cardiac camera. J Nucl Cardiol 2016;23:514-26.

11. Daou D, Sabbah R, Coaguila C, Boulahdour H. Feasibility of datadriven cardiac respiratory motion correction of myocardial perfusion CZT SPECT: a pilot study. J Nucl Cardiol 2016;2016:1-10.
12. Buechel RR, Pazhenkottil AP, Herzog BA, Husmann L, Nkoulou $\mathrm{RN}$, Burger IA, et al. Real-time breath-hold triggering of myocardial perfusion imaging with a novel cadmium-zinc-telluride detector gamma camera. Eur $\mathrm{J}$ Nucl Med Mol Imaging 2010;37:1903-8.

13. Buechel RR, Husmann L, Pazhenkottil AP, Nkoulou R, Herzog BA, Burger IA, et al. Myocardial perfusion imaging with real-time respiratory triggering: impact of inspiration breath-hold on left ventricular functional parameters. J Nucl Cardiol 2010;17:848-52.

14. Clerc OF, Fuchs TA, Possner M, Vontobel J, Mikulicic F, Stehli J et al. Real-time respiratory triggered SPECT myocardial perfusion imaging using CZT technology: impact of respiratory phase matching between SPECT and low-dose CT for attenuation correction. Eur Heart J Cardiovasc Imaging 2016. doi:10.1093/ ehjci/jew031.

15. Christou MA, Siontis GC, Katritsis DG, Ioannidis JP. Meta-analysis of fractional flow reserve versus quantitative coronary angiography and noninvasive imaging for evaluation of myocardial ischemia. Am J Cardiol 2007;99:450-6.

16. Melikian N, De Bondt P, Tonino P, De Winter O, Wyffels E, Bartunek J, et al. Fractional flow reserve and myocardial perfusion imaging in patients with angiographic multivessel coronary artery disease. JACC Cardiovasc Interv 2010;3:307-14.

17. Mouden M, Ottervanger JP, Knollema S, Timmer JR, Reiffers S, Oostdijk AH, et al. Myocardial perfusion imaging with a cadmium zinc telluride-based gamma camera versus invasive fractional flow reserve. Eur J Nucl Med Mol Imaging 2014;41:956-62.

18. Fitzgerald J, Danias PG. Effect of motion on cardiac SPECT imaging: recognition and motion correction. J Nucl Cardiol 2001;8:701-6.

19. van Dijk JD, van Dalen JA, Mouden M, Ottervanger JP, Knollema $\mathrm{S}$, Slump $\mathrm{CH}$, et al. Value of automatic patient motion detection and correction in myocardial perfusion imaging using a CZTbased SPECT camera. J Nucl Cardiol. 2016. doi:10.1007/s12350016-0571-7. 